\title{
Cargo trafficking between endosomes and the trans-Golgi network
}

Pei Zhi Cheryl Chia", Pricilla Gunn and Paul A. Gleeson

*The Department of Biochemistry and Molecular Biology and Bio21 Molecular Science and Biotechnology Institute, The University of Melbourne, Victoria 3010, Australia

(\# current address; Mucosal Immunology Section, National Institutes of Health, Bethesda, USA)

Correspondence should be addressed to:

Professor Paul Gleeson

Department of Biochemistry and Molecular Biology and Bio21 Molecular Science and Biotechnology

Institute

The University of Melbourne

Melbourne, Victoria 3010

Australia

Phone 61-3-8344-2354: $\quad$ Fax $\quad 61-3-9348-1428$

E-mail:pgleeson@unimelb.edu.au 


\begin{abstract}
The retrograde membrane transport pathways from endosomes to the trans-Golgi network are now recognised as critical intracellular pathways to recycle and shuttle a selective subgroup of membrane proteins, including sorting receptors, membrane bound enzymes, transporters, as well as providing an avenue for the intracellular transport of various bacterial toxins. Multiple pathways from endosomes to the TGN have now been defined which differ in the cargo transported and the machinery used. Here we review advances in these pathways and the requirement for TGN organisation, and also discuss the development of unbiased analytical approaches to quantitatively track cargo that use these endosome-to-TGN pathways.
\end{abstract}

Keywords: trans-Golgi network, retrograde transport, membrane trafficking, membrane recycling, endosomes 


\section{Introduction}

The trans-Golgi network (TGN) is a key organelle for protein transport in all eukaryotic cells. The TGN is the exit face of the Golgi and acts as a major traffic hub regulating membrane transport in the cell's secretory pathway as well as receiving protein cargo from endosome compartments (socalled 'retrograde' transport). Bidirectional traffic between the TGN and the endosomal system is essential to the functional integrity of the Golgi apparatus and more broadly, for development, organelle biogenesis, and nutrient homeostasis (Burd 2011; Johannes and Popoff 2008; Pavelka et al. 2008). Furthermore, many bacterial toxins mediate cytotoxicity and some viral proteins promote pathogenicity by exploitation of endosome-to-TGN pathways.

Despite the central role of transport pathways between the TGN and endosomes in cell and developmental biology, these pathways have received less attention compared with endoplasmic reticulum-to-Golgi transport and clathrin-mediated endocytosis at the plasma membrane (PM). However, recent studies have begun to resolve these pathways in detail, define the molecular machinery for each pathway, and the choice of pathway for an increasing range of different cargo.

A prime function of retrograde transport is to retrieve protein cargo from the plasma membrane and endosomes for reuse. Anterograde transport from the Golgi results in the depletion of sorting receptors, processing enzymes, and vesicle targeting and fusion factors, all of which must be returned to the TGN to maintain its function. Retrograde transport from the endosomes to the TGN is important in many physiological and cellular processes in flies, worms and mammals (Bonifacino and Rojas 2006a; Burd 2011; Johannes and Popoff 2008). For example, regulation of the secretion of Wnt signalling factors by the sorting receptor Wntless is dependent on the continuous recycling and retrograde transport of Wntless (Gasnereau et al. 2011).

Several retrograde transport pathways from different endosomal compartments to the TGN have now been identified (Bonifacino and Rojas 2006a; Johannes and Popoff 2008; Lieu and Gleeson 2011) (Fig 1). These include pathways from the early and recycling endosomes to the TGN and from the late endosomes to the TGN (Lieu and Gleeson 2011). Multicellular organisms have a greater complexity in their retrograde transport pathways than single cell eukaroytes such as yeast 
that are often used as a model system (Bonifacino and Rojas 2006a) and this complexity is reflected by a greater number of regulatory components that regulate the pathways in multicellular organisms. The evolution of these pathways is likely to stem from their central roles in the development and physiology of multicellular organisms. This review will focus on some recent advances of the pathways involved and the emergence of a new method to quantitatively track the movement of cargo along endosome-to-TGN pathways.

\section{Retrograde pathway cargo}

The retrograde transport route is used to transport a highly selective subgroup of membrane proteins. The cargo proteins that use this pathway have a variety of functions and can be classified broadly into four groups: (i) sorting receptors such as mannose-6-phosphate receptors (MPRs) and Wntless, (ii) integral membrane proteases such as -secretase, furin and the yeast Kex2, (iii) SNAREs and (iv) nutrient and ion transporters (Bonifacino and Rojas 2006a; Ghosh et al. 2003; Lewis et al. 2000; Shewan et al. 2003). In addition, bacterial and plant toxins such as Shiga toxin, cholera toxin and ricin, are internalized by endocytosis and then undergo retrograde transport to the TGN as an essential step in mediating cytotoxicity (Sandvig and van Deurs 2000; Sandvig et al. 2013; Utskarpen et al. 2006).

The membrane protein TGN38/42 and the non-toxic receptor-binding B-subunit of bacterial Shiga toxin (SxTB) have been used as model cargo to examine retrograde transport and have played key roles in the discovery and dissection of membrane trafficking at the early/recycling endosome-TGN interface (Johannes and Popoff 2008; Lieu and Gleeson 2011). With the use of intracellular markers, these pathways can be broadly divided into retrograde transport from the recycling endosomes, the early endosomes or the late endosomes, to the TGN. Each pathway is characterized by distinct sets of sorting machinery and the retrograde cargo that utilize these pathways. However, some overlap in machinery exists between pathways, in part a reflection of the complexity of the nature of endosomal compartments.

Endosomes are a complex membrane system of tubular-vacuolar nature of which the early endosomes and late endosomes/lysosomes are the major components (Gruenberg and Stenmark 2004). The endocytic recycling compartment and associated recycling endosomes are often perceived as a differentiated subdomain of the tubular endosomal network (Bonifacino and Rojas 
2006b) or extensions of the early endosomes and in fact, cannot be distinguished from the early endosome by any one protein or lipid, apart from morphological differences (Maxfield and McGraw 2004). To compound the problem, early endosomes and late endosomes may also represent a continuum rather than distinct compartments. For some time, endosomal cargo sorting was based on the notion of stable compartments between which cargo traffic in vesicular intermediates. Each compartment is thus assumed to have a defined and constant composition, and function as a distinct, long-lived unit in recycling. However, there has been a gradual shift in view, to one where endosomes are thought to undergo gradual maturation from nascent early endosomes to maturing early endosomes, to early late endosomes and then mature late endosomes. The composition of endosomal membranes at different stages of this continuum can be imagined as a 'mosaic', with various levels of proteins or lipids rather than an absolute presence of absence of a particular marker (Cullen 2008).

Thus, the division of retrograde pathways into three main routes may be an oversimplification of the complexity of transport processes between the endo-lysosomal system and the TGN. Nonetheless, the retrograde routes and cargoes that utilize these pathways highlighted below have been instrumental in augmenting our understanding of retrograde transport processes.

\section{The early endosome-to-TGN pathway}

Mallard et al. (Mallard et al. 1998) provided the first evidence for the early endosome-to-TGN (EEto-TGN) route, using STxB as a model cargo. Clathrin was found to be required for exit of STxB from early endosomes (Saint-Pol et al. 2004). Some clathrin adaptors are also involved in retrograde transport of STxB, such as AP1 (Meyer et al. 2000), epsinR (Saint-Pol et al. 2004) and OCRL (oculocerebrorenal syndrome of Lowe) (Choudhury et al. 2005), the last of which is a $\mathrm{PI}(4,5) \mathrm{P}_{2}$-phosphatase that interacts with the clathrin heavy chain and promotes clathrin assembly in vitro. MPRs may also traffic directly from early endosomes to the TGN, as MPRs accumulate in early endosomes under many conditions that block retrograde transport to the TGN, such as perturbations in AP1 (Meyer et al. 2000) and PACS-1 (Scott et al. 2006) and PIKfyve (Rutherford et al. 2006).

The retromer complex is another important regulator of retrograde transport that is localized on early endosomes and has been shown to be required for retrograde transport of both MPRs and STxB (Bonifacino and Hurley 2008). The finding that clathrin components and retromer are both 
needed for MPRs and STxB have led many to suggest that they may be functionally linked (Johannes and Popoff, 2008). A combined action may be mediated by proteins that link retromer and clathrin at the early endosomes, such as Hrs (Popoff et al. 2009; Chin et al. 2001). In addition, the early endosomal $\mathrm{J}$ domain protein RME-8 recruits SNX1 and the clathrin uncoating ATPase Hsc70. This simultaneous interaction may thus couple the recruitment of the retromer with the shedding of the clathrin coat (Popoff et al. 2009; Shi et al. 2009).

Specific tethering machinery and SNARE complexes have been associated with EE-to-TGN transport. In particular, the TGN golgin GCC88 appears to play a role in EE-to-TGN transport. Surprisingly, GCC88-depletion resulted in the retention of CI-MPR and TGN38 at the early endosomes, but did not perturb retrograde transport of STxB (Lieu et al. 2007). This indicates that there may be some distinction between the retrograde pathways of STxB and TGN38, or that the TGN golgins may add another level of specificity to retrograde sorting (Lieu et al. 2007; Lieu and Gleeson 2010). In addition, the GARP complex was shown to be required for the tethering of MPRpositive vesicles to the TGN membranes (Perez-Victoria et al. 2008). GARP is recruited to the TGN by the TGN-localized Rab6 GTPase (Liewen et al. 2005) and is thought to promote SNARE complex formation. Two SNARE complexes have been implicated in transport from early/recycling endosomes to the TGN: the syntaxin 6/syntaxin 16/Vtila/VAMP3 or VAMP4 SNARE complex and the syntaxin 5/GS15/GS28/Ykt6 SNARE complex (Mallard et al. 2002; Amessou et al. 2007; Xu et al. 2002; Tai et al. 2004).

\section{The late endosome-to-TGN pathway}

Pfeffer and colleagues were the first to propose that MPRs recycle to the TGN via the late endosomes (Lombardi et al. 1993). A number of machinery components have been shown to be specifically involved in the late endosome-to-TGN (LE-to-TGN) transport of MPRs, such TIP47 and Rab9 (Carroll et al. 2001; Lombardi et al. 1993). Rab9 can also bind the TGN golgin GCC185 (Reddy et al. 2006). Surprisingly, depletion of GCC185 perturbs retrograde transport of not only MPRs but also STxB (Derby et al. 2007), suggesting that GCC185 regulates more than one retrograde transport pathway. In support of this proposal, GCC185 also interacts with Rab6 and syntaxin 16, both of which are involved in EE-to-TGN trafficking (Mallard et al. 2002).

Transport of MPRs from Rab9-positive late endosomes to the TGN is dependent on a specific syntaxin 10/syntaxin 16/Vtila/VAMP3 SNARE complex (Wang et al. 2005; Ganley et al. 2008). Depletion of syntaxin 10 perturbed retrograde transport of MPRs but had no effect on that of the 
EE-to-TGN transport of TGN46 and cholera toxin (Ganley et al. 2008). In contrast, depletion of syntaxin 6, which is part of the SNARE complex involved in EE-to-TGN transport, disrupted retrograde transport of TGN46 and cholera toxin (Ganley et al. 2008). Thus the syntaxin 10SNARE complex appears to function exclusively along LE-to-TGN transport.

Furin is a membrane proprotein convertase, whose primary role is to cleave the proprotein domains of immature proteins to trigger their enzymatic activation (Thomas 2002). Our studies have recently shown that furin utilizes the retrograde route leading from late endosomes to the TGN and is dependent on the late endosome-localized Rab9 GTPase, the TGN golgin GCC185 and the tSNARE syntaxin 10 for efficient TGN-retrieval (Chia et al. 2011). Furin is only the second cargo to be found to use the LE-to-TGN retrograde pathway and indeed many parallels can be found between the trafficking of furin and CI-MPR, in terms of their dependence on the specific trafficking machinery, Rab9, TGN golgin GCC185, and syntaxin 10 (Chia et al. 2011).

\section{The recycling endosome-to-TGN pathway}

Relatively few cargos have been shown to use the recycling endosome-to-TGN (RE-to-TGN) route, perhaps due to that fact that very few exclusive markers for this compartment exist. Early studies by Maxfield and colleagues using a Tac-TGN38 chimera showed that the chimeric protein was delivered to the recycling compartment en route to the TGN (Ghosh et al. 1998). However, further studies showed that full-length TGN38 utilized a direct EE-to-TGN route instead (Lieu et al. 2007; Banting and Ponnambalam 1997). This discrepancy may be explained by the use of the transferrin receptor as a marker of the recycling endosomes in early experiments, which has an itinerary involving both early and recycling endosomes. STxB has also proposed to use the RE-to-TGN pathway, based on the fact that GCC185-depletion resulted in retention of STxB in Rab11-positive recycling endosomes (Lieu and Gleeson 2010). This finding was perhaps difficult to reconcile with the established role of retromer, thought to act at a point between early and late endosomes. However, a recent report by McKenzie and colleagues, utizing ablation of recycling endosomes, has suggested that retromer is required also for early endosome to recycling endosome transport step and that both CI-MPR and Shiga toxin pass through the recycling endosomes to the Golgi ((McKenzie et al. 2012).

Another cargo that has been shown to undergo retrograde transport from the early and recycling endosomes, is the v-SNARE VAMP4 (Tran et al. 2007). This recycling route is consistent with the role of VAMP4 in mediating retrograde transport of cargoes from early/recycling endosomes, 
through formation of a SNARE complex with syntaxin 6, syntaxin 16 and Vtila (Mallard et al. 2002).

\section{Retrograde transport in specialized cells with fragmented Golgi}

The majority of studies on retrograde transport have been carried out using immortalized cells where the mammalian Golgi is organized as a ribbon structure during interphase of the cell cycle. Given the microtubule-dependent process of retrograde transport to the perinuclear location of the Golgi ribbon (Wassmer et al. 2009), the question arises whether the Golgi needs to be organized in a continuous ribbon structure for functional retrograde transport to occur. This question is relevant as there is considerable plasticity in the arrangement of the Golgi stacks and the Golgi apparatus deviates from a ribbon conformation in a variety of cell processes. For example, in mammalian cells regulated fragmentation of the Golgi occurs during mitosis (Colanzi et al. 2003; Nelson 2000) and repositioning of the Golgi can occur during a number of processes, including directed secretion and pathogen invasion (Kupfer et al. 1983; Yadav et al. 2009). Moreover the structure of the Golgi differs in different cell types. The Golgi of plant cells is not a ribbon structure, rather the Golgi consists of very large number of individual Golgi stacks (Faso et al. 2009; Hawes et al. 2010). In yeast cells the Golgi has been identified as either ordered individual stacks, as in Pichia pastoris, or individual cisternae spread throughout the cytoplasm (Papanikou and Glick 2009). Drosophila imaginal disk cells have dispersed Golgi mini-stacks (Yano et al. 2005).

We recently discovered that mouse gastric parietal cells, a highly differentiated cell of the gastric mucosa, does not have a Golgi ribbon rather the Golgi apparatus in these cells is dispersed as ministacks throughout the cytoplasm (Gunn et al. 2011) (Figure 2). The highly specialized parietal cell is an epithelial cell that secretes acid into the lumen of the stomach (Pavelka and Roth 2010). The dispersed Golgi stacks of cultured parietal cells were shown to function in both anterograde and retrograde membrane transport (Gunn et al. 2011). Of particular relevance to this review is the trafficking of the model cargo cholera toxin $(\mathrm{ChxB})$ which can undergo retrograde transport from the plasma membrane to the Golgi in other cells. Quantitation of cholera toxin after $4 \mathrm{hr}$ internalization revealed that approximately $25 \%$ of total $\mathrm{ChxB}$ was co-localized with the marker Golgi GM130, demonstrating that plasma membrane-to-Golgi transport pathway is functional in parietal cells and that the majority of Golgi mini-stacks can receive cargo via retrograde transport (Gunn et al. 2011). These findings demonstrated a functional retrograde transport pathway in differentiated cells with a highly dispersed and fragmented Golgi apparatus. Given the 
extraordinary abundance of $\mathrm{H} / \mathrm{K}$ ATPase containing specialized membranes (tubulovesicular elements) in the cytoplasm of parietal cells (Scarff et al. 1999), the scattered Golgi stacks may provide a more efficient mechanism for delivery of retrograde transport carriers compared with a location deeper in the perinuclear region of the cell.

\section{Sorting into the retrograde pathway: potential role of transmembrane domain}

Although the machinery of transport for each of the retrograde transport pathways is becoming increasingly well-defined, less is known about the identity of the sorting signals for retrieval of cargo proteins from lysosomal degradation into the specific retrograde transport routes. Discrete sorting motifs in the cytoplasmic tails of recycling proteins have been shown to be required for the endocytosis and TGN-retrieval of cargo (Bonifacino and Traub 2003). For example, acidic cluster motifs in the cytoplasmic tail of furin interacts with the adaptor phosphofurin acidic cluster sorting protein-1 (PACS-1) (Wan et al. 1998), an aromatic-containing sorting motif found in the tail of M6P-R and sortilin interacts with retromer (Seaman 2007; Tabuchi et al. 2010) and a FW or P-rich motif in the tail of M6P-R is important for interaction with TIP47 (Diaz and Pfeffer 1998; Krise et al. 2000). However, signals in addition to those in the cytoplasmic tails may also be required. Sorting of membrane proteins from the early endosomes for direct transport to the Golgi requires the segregation of cargo from the vacuolar membrane of the endosome into the tubular endosomal extensions from which transport carriers are derived (Bonifacino and Rojas 2006a; Cullen 2008; Cullen and Korswagen 2012). Our work has shown that TGN38 is transported directly from early endosomes to the TGN whereas furin is transported via the Rab9-dependent retrograde pathway from the late endosomes (Chia et al. 2011; Lieu and Gleeson 2010). We have recently exploited the distinct retrograde transport pathways of these two cargos to dissect the sorting signals for their segregation within early endosome. Our surprising discovery is that the segregation of the two cargo at the early endosome is dependent on both the transmembrane domain (TMD) and the cytoplasmic tail. Based on these finding (Chia et al. 2011), we have proposed that two signals are required to enter the retrograde pathway from the early endosome, firstly a TMD which is compatible with the highly mobile disordered lipid domains of the tubules, and secondly, specific motifs in the cytoplasmic tail which can interact with protein coats such as retromer (Cullen and Korswagen 2012).

The early endosome can be divided into a highly tubulated subregion and the main body/limiting membrane that encompasses the intraluminal vesicles (Gruenberg, 2001). The morphological 
heterogeneity of early endosomal subdomains may be attributable to differences in lipid composition. By generating tubules from giant unilaminar vesicles with different lipid compositions, Goud and colleagues (Roux et al., 2005) were able to show that rigid lipids like cholesterol and sphingomyelin were excluded from tubular regions. Thus, it is possible that the more tubular regions of the early endosome have a lower level of saturated lipids, consistent with the high level of curvature required for tubulation. On the other hand, the concentration of such lipids in the limiting membrane would result in thicker bilayers with less curvature. However, confirming a difference in lipid composition between subregions of one organelle is technically difficult, and so far has only been achieved for whole membranes of organelles such as the Golgi or the PM (Graham 2001; Lee et al. 2008). TMDs assume an $\alpha$-helical structure in lipid bilayer and a difference of 2-3 residues would equate to an increase in 3-4.5 Angstroms in length. Whether such a difference is biologically relevant will depend on the relative thickness of endosomal membranes.

Nonetheless, the existence of distinct early endosomal subdomains could explain how TMD length can influence sorting events. According to the general model of protein-lipid sorting proposed by Munro (Bretscher and Munro 1993; Sharpe et al. 2010) proteins with shorter TMD lengths may be preferentially segregated into subregions with thinner bilayers and more tubular profiles. The clustering of these cargoes would result in the recruitment of trafficking machinery such as retromer, via retromer-interacting motifs, to the tubular subregions of the early endosome, facilitating efficient transport of cargoes out of this compartment. On the other hand, proteins with longer TMDs would remain in the thicker bilayers of the limiting membrane, and are retained in the main body of the early endosome as it matures into a late endosome. Cargos lacking TGN-retrieval motifs would be unable to exit the maturing endosome and are ultimately delivered to the lysosomes.

\section{New methods for monitoring intracellular cargo transport using flow cytometry}

In spite of the recent progress in mapping endosome-to-TGN pathways, a major limitation in accurately defining the retrograde transport of cargo is the capacity to quantitatively track the intracellular movement of cargo. The availability of methods for high-throughput quantitative analyses of cargo trafficking in cell populations would overcome one major limitation and be a widely applicable advance in the field. 
Standard approaches for detecting the intracellular location of cargo involves microscopy-based methods that are highly limited in their throughput capacity, speed of acquisition and quantitation. Typically 25-50 cells are analyzed by immunofluorescence for each condition, a time consuming process. Furthermore the acquisition of 2D images creates huge data files and necessitates complex data analysis strategies. The development of fast, sensitive and quantitative approaches to detect intracellular localization and trafficking would represent a major advance in the field. Flow cytometry has the capacity to analyze thousands of cells per second. The classical use of FACS has involved collection of the total fluorescence emitted by each cell, eg. pulse area, as it pass through the laser light beam, rather than using the instrument to quantitate different intracellular locations. FACS analyses are usually represented as pulse area (Figure 3A). In contrast, pulse width provides information about the size of the fluorescent structure. Pulse width has been used in the past to discriminate a pulse due to a single cell from a pulse from a doublet (two cells stuck together) (Wersto et al. 2001). However, pulse width can be used for other purposes other than doublet discrimination. We have recently described pulse shape analysis (PulSA) to monitor protein localization changes in cells by flow cytometry (Ramdzan et al. 2012) (Figure 3B). We assessed the applicability of PulSA for monitoring protein localization differences on a number of biological processes including the internalization and retrograde trafficking of plasma membrane receptorbound proteins to the Golgi using STxB. The pulse width of fluorescently labelled STxB shifted to match its cellular location, as confirmed by confocal microscopy (Ramdzan et al. 2012) (Chia et al unpublished observations). Major changes in pulse width measurements demonstrated that PulSA specifically tracks protein localization changes and that these effects do not arise from changes in cell or organelle morphology. With flow cytometry flow rates of $>10,000$ cells per sec, and availability of 8-colour analyses, PulSA provides a considerable advance in quantitative analysis of cargo trafficking.

\section{Concluding remarks}

Two main routes for the recycling of proteins have been identified between the cell surface and intracellular compartments. Membrane proteins can undergo recycling between the cell surface and endosomes, which is a feature of proteins such as transferrin receptor, whilst other proteins recycle between the plasma membrane and the TGN, and as a result, require retrograde transport from the endosomes to the TGN. A number of retrograde pathways leading from the endosomes to the TGN have now been identified (Johannes and Popoff, 2008; Lieu and Gleeson, 2011). These include 
transport pathways from the early and recycling endosomes to the TGN, used by TGN38 and Shiga toxin, respectively and a pathway from the late endosome to the TGN, used by the MPRs and furin.

A key question that arises from examining the different itineraries of recycling proteins is the biological basis for a specific recycling pathway. Clearly a physiological basis must exist to expain the trafficking along a certain retrograde route. In the case of CI-MPR, prolonged retention in maturing endosomes is important for the release of bound acid-hydrolases at the low $\mathrm{pH}$ of the late endosome, before recycling of CI-MPR to the TGN for further rounds of sequestration. However, the basis for the retrograde transport route for furin is not as obvious. One possibility is that furin may require retention in maturing endosomes for the efficient cleavage and activation of as yet unidentified endogenous substrates within endosomal compartments. If this is the case then diversion of furin along the early endosome-to-TGN pathway could result in insufficient cleavage of endosomal substrates. Manipulation of the retrograde sorting signals of furin so that it is recycled to the TGN directly from the early endosomes and bypasses the late endosomal compartment (Chia et al. 2011), will allow physiological impact of directing furin along a different retrograde route to be explored in cells and organisms.

The application of a suite of imaging technologies and in particular the marriage of flow cytometry and imaging has considerable potential to enhance the capacity to rapidly analyse Golgi trafficking events in large number of cells in a unbiased manner. In addition, the use of flow cytometry for analysis of protein location has considerable promise for the assessment of the impact of membrane perturbation reagents and specific inhibitors on Golgi trafficking and the isolation of mutants defective in these processes.

In summary, with the advances in approaches to monitor trafficking pathway and knowledge of the machinery and cargo sorting signals, there is now considerable potential to further probe the importance of membrane trafficking events from endosomal compartments to the TGN in different physiological systems and organisms.

\section{Acknowledgements}

This work was supported by funding from the Australian Research Council, National Health \& Medical Research Council of Australia 


\section{References}

Amessou M, Fradagrada A, Falguieres T, Lord JM, Smith DC, Roberts LM, Lamaze C, Johannes L (2007) Syntaxin 16 and syntaxin 5 are required for efficient retrograde transport of several exogenous and endogenous cargo proteins. J Cell Sci 120 (Pt 8):1457-1468

Banting G, Ponnambalam S (1997) TGN38 and its orthologues: roles in post-TGN vesicle formation and maintenance of TGN morphology. Biochim Biophys Acta 1355 (3):209-217. doi:S0167-4889(96)00146-2 [pii]

Bonifacino JS, Hurley JH (2008) Retromer. Curr Opin Cell Biol 20 (4):427-436. doi:S09550674(08)00043-4 [pii] 10.1016/j.ceb.2008.03.009

Bonifacino JS, Rojas R (2006a) Retorgrade transport from endosomes to the trans-Golgi network. Nature Rev Mol Cell Biol 7:568-579

Bonifacino JS, Rojas R (2006b) Retrograde transport from endosomes to the trans-Golgi network. Nat Rev Mol Cell Biol 7 (8):568-579. doi:nrm1985 [pii] 10.1038/nrm1985

Bonifacino JS, Traub LM (2003) Signals for sorting of transmembrane proteins to endosomes and lysosomes. Annu Rev Biochem 72:395-447. doi:10.1146/annurev.biochem.72.121801.161800 121801.161800 [pii]

Bretscher MS, Munro S (1993) Cholestrol and Golgi apparatus. Science 261:1280-1281

Burd CG (2011) Physiology and pathology of endosome-to-Golgi retrograde sorting. Traffic 12 (8):948-955. doi:10.1111/j.1600-0854.2011.01188.x

Carroll KS, Hanna J, Simon I, Krise J, Barbero P, Pfeffer SR (2001) Role of Rab9 GTPase in facilitating receptor recruitment by TIP47. Science 292 (5520):1373-1376

Chia PZ, Gasnereau I, Lieu ZZ, Gleeson PA (2011) Rab9-dependent retrograde transport and endosomal sorting of the endopeptidase furin. J Cell Sci. doi:jcs.083782 [pii] $10.1242 / \mathrm{jcs} .083782$

Chin LS, Raynor MC, Wei X, Chen HQ, Li L (2001) Hrs interacts with sorting nexin 1 and regulates degradation of epidermal growth factor receptor. J Biol Chem 276 (10):7069-7078. doi:10.1074/jbc.M004129200 M004129200 [pii]

Choudhury R, Diao A, Zhang F, Eisenberg E, Saint-Pol A, Williams C, Konstantakopoulos A, Lucocq J, Johannes L, Rabouille C, Greene LE, Lowe M (2005) Lowe syndrome protein OCRL1 interacts with clathrin and regulates protein trafficking between endosomes and the trans-Golgi network. Mol Biol Cell 16 (8):3467-3479

Colanzi A, Suetterlin C, Malhotra V (2003) Cell-cycle-specific Golgi fragmentation: how and why? Curr Opin Cell Biol 15 (4):462-467. doi:S095506740300067X [pii] 
Cullen PJ (2008) Endosomal sorting and signalling: an emerging role for sorting nexins. Nat Rev Mol Cell Biol 9 (7):574-582

Cullen PJ, Korswagen HC (2012) Sorting nexins provide diversity for retromer-dependent trafficking events. Nature Cell Biology 14 (1):29-37. doi:10.1038/ncb2374

Derby MC, Lieu ZZ, Brown D, Stow JL, Goud B, Gleeson PA (2007) The trans-Golgi Network Golgin, GCC185, is Required for Endosome-to-Golgi Transport and Maintenance of Golgi Structure. Traffic 8 (6):758-773

Diaz E, Pfeffer SR (1998) TIP47: a cargo selection device for mannose 6-phosphate receptor trafficking. Cell 93 (3):433-443

Faso C, Boulaflous A, Brandizzi F (2009) The plant Golgi apparatus: last 10 years of answered and open questions. FEBS Lett 583 (23):3752-3757. doi:S0014-5793(09)00746-7 [pii] 10.1016/j.febslet.2009.09.046

Ganley IG, Espinosa E, Pfeffer SR (2008) A syntaxin 10-SNARE complex distinguishes two distinct transport routes from endosomes to the trans-Golgi in human cells. J Cell Biol 180 (1):159-172

Gasnereau I, Herr P, Chia PZ, Basler K, Gleeson PA (2011) Identification of an endocytosis motif in an intracellular loop of Wntless protein, essential for its recycling and the control of Wnt protein signaling. The Journal of biological chemistry 286 (50):43324-43333. doi:10.1074/jbc.M111.307231

Ghosh P, Dahms NM, Kornfeld S (2003) Mannose 6-phosphate receptors: new twists in the tale. Nat Rev Mol Cell Biol 4 (3):202-212

Ghosh RN, Mallet WG, Soe TT, McGraw TE, Maxfield FR (1998) An endocytosed TGN38 chimeric protein is delivered to the TGN after trafficking through the endocytic recycling compartment in CHO cells. J Cell Biol 142 (4):923-936

Graham JM (2001) Isolation of Golgi membranes from tissues and cells by differential and density gradient centrifugation. Curr Protoc Cell Biol Chapter 3:Unit 39. doi:10.1002/0471143030.cb0309s10

Gruenberg J, Stenmark H (2004) The biogenesis of multivesicular endosomes. Nat Rev Mol Cell Biol 5 (4):317-323. doi:10.1038/nrm1360 nrm1360 [pii]

Gunn PA, Gliddon BL, Londrigan SL, Lew AM, van Driel IR, Gleeson PA (2011) The Golgi apparatus in the endomembrane-rich gastric parietal cells exist as functional stable ministacks dispersed throughout the cytoplasm. Biology of the cell / under the auspices of the European Cell Biology Organization 103 (12):559-572. doi:10.1042/BC20110074 
Hawes C, Schoberer J, Hummel E, Osterrieder A (2010) Biogenesis of the plant Golgi apparatus. Biochemical Society transactions 38 (3):761-767. doi:BST0380761 [pii] 10.1042/BST0380761

Johannes L, Popoff V (2008) Tracing the retrograde route in protein trafficking. Cell 135 (7):11751187

Krise JP, Sincock PM, Orsel JG, Pfeffer SR (2000) Quantitative analysis of TIP47-receptor cytoplasmic domain interactions: implications for endosome-to-trans Golgi network trafficking. The Journal of biological chemistry 275 (33):25188-25193. doi:10.1074/jbc.M001138200

Kupfer A, Dennert G, Singer SJ (1983) Polarization of the Golgi apparatus and the microtubuleorganizing center within cloned natural killer cells bound to their targets. Proc Natl Acad Sci U S A 80 (23):7224-7228

Lee YC, Block G, Chen H, Folch-Puy E, Foronjy R, Jalili R, Jendresen CB, Kimura M, Kraft E, Lindemose S, Lu J, McLain T, Nutt L, Ramon-Garcia S, Smith J, Spivak A, Wang ML, Zanic M, Lin SH (2008) One-step isolation of plasma membrane proteins using magnetic beads with immobilized concanavalin A. Protein Expr Purif 62 (2):223-229. doi:S1046-5928(08)00215-5 [pii] 10.1016/j.pep.2008.08.003

Lewis MJ, Nichols BJ, Prescianotto-Baschong C, Riezman H, Pelham HR (2000) Specific retrieval of the exocytic SNARE Snc1p from early yeast endosomes. Mol Biol Cell 11 (1):23-38

Lieu ZZ, Derby MC, Teasdale RD, Hart C, Gunn P, Gleeson PA (2007) The golgin GCC88 is required for efficient retrograde transport of cargo from the early endosomes to the transGolgi network. Mol Biol Cell 18 (12):4979-4991. doi:E07-06-0622 [pii]

10.1091/mbc.E07-06-0622

Lieu ZZ, Gleeson PA (2010) Identification of different itineraries and retromer components for endosome-to-Golgi transport of TGN38 and Shiga toxin. Eur J Cell Biol 89 (5):379-393. doi:S0171-9335(10)00009-9 [pii] 10.1016/j.ejcb.2009.10.021

Lieu ZZ, Gleeson PA (2011) Endosome-to-Golgi transport pathways in physiological processes. Histol Histopathol 26 (3):395-408

Liewen H, Meinhold-Heerlein I, Oliveira V, Schwarzenbacher R, Luo G, Wadle A, Jung M, Pfreundschuh M, Stenner-Liewen F (2005) Characterization of the human GARP (Golgi associated retrograde protein) complex. Exp Cell Res 306 (1):24-34

Lombardi D, Soldati T, Riederer MA, Goda Y, Zerial M, Pfeffer SR (1993) Rab9 functions in transport between late endosomes and the trans Golgi network. Embo J 12 (2):677-682 
Mallard F, Antony C, Tenza D, Salamero J, Goud B, Johannes L (1998) Direct pathway from early/recycling endosomes to the Golgi apparatus revealed through the study of shiga toxin Bfragment transport. J Cell Biol 143 (4):973-990

Mallard F, Tang BL, Galli T, Tenza D, Saint-Pol A, Yue X, Antony C, Hong W, Goud B, Johannes L (2002) Early/recycling endosomes-to-TGN transport involves two SNARE complexes and a Rab6 isoform. J Cell Biol 156 (4):653-664

Maxfield FR, McGraw TE (2004) Endocytic recycling. Nat Rev Mol Cell Biol 5 (2):121-132. doi: $10.1038 / \mathrm{nrm} 1315$

McKenzie JE, Raisley B, Zhou X, Naslavsky N, Taguchi T, Caplan S, Sheff D (2012) Retromer guides STxB and CD8-M6PR from early to recycling endosomes, EHD1 guides STxB from recycling endosome to Golgi. Traffic 13 (8):1140-1159. doi:10.1111/j.16000854.2012.01374.x

Meyer C, Zizioli D, Lausmann S, Eskelinen EL, Hamann J, Saftig P, von Figura K, Schu P (2000) mu1A-adaptin-deficient mice: lethality, loss of AP-1 binding and rerouting of mannose 6phosphate receptors. EMBO J 19 (10):2193-2203. doi:10.1093/emboj/19.10.2193

Nelson WJ (2000) W(h)ither the Golgi during mitosis? Journal of Cell Biology 149 (2):243-248 Papanikou E, Glick BS (2009) The yeast Golgi apparatus: insights and mysteries. FEBS Lett 583 (23):3746-3751. doi:S0014-5793(09)00864-3 [pii] 10.1016/j.febslet.2009.10.072

Pavelka M, Neumuller J, Ellinger A (2008) Retrograde traffic in the biosynthetic-secretory route. Histochemistry and cell biology 129 (3):277-288. doi:10.1007/s00418-008-0383-1

Pavelka M, Roth J (2010) Functional ultrastructure. An atlas of tissue biology and pathology. Springer, Vienna, New York

Perez-Victoria FJ, Mardones GA, Bonifacino JS (2008) Requirement of the human GARP complex for mannose 6-phosphate-receptor-dependent sorting of cathepsin D to lysosomes. Mol Biol Cell 19 (6):2350-2362. doi:E07-11-1189 [pii] 10.1091/mbc.E07-11-1189

Popoff V, Mardones GA, Bai SK, Chambon V, Tenza D, Burgos PV, Shi A, Benaroch P, Urbe S, Lamaze C, Grant BD, Raposo G, Johannes L (2009) Analysis of articulation between clathrin and retromer in retrograde sorting on early endosomes. Traffic 10 (12):1868-1880. doi:TRA993 [pii] 10.1111/j.1600-0854.2009.00993.x

Ramdzan YM, Polling S, Chia CP, Ng IH, Ormsby AR, Croft NP, Purcell AW, Bogoyevitch MA, Ng DC, Gleeson PA, Hatters DM (2012) Tracking protein aggregation and mislocalization in cells with flow cytometry. Nat Methods 9 (5):467-470. doi:10.1038/nmeth.1930 nmeth.1930 [pii] 
Reddy JV, Burguete AS, Sridevi K, Ganley IG, Nottingham RM, Pfeffer SR (2006) A functional role for the GCC185 golgin in mannose 6-phosphate receptor recycling. Mol Biol Cell 17 (10):4353-4363

Rutherford AC, Traer C, Wassmer T, Pattni K, Bujny MV, Carlton JG, Stenmark H, Cullen PJ (2006) The mammalian phosphatidylinositol 3-phosphate 5-kinase (PIKfyve) regulates endosome-to-TGN retrograde transport. J Cell Sci 119 (Pt 19):3944-3957. doi:jcs.03153 [pii] $10.1242 /$ jcs. 03153

Saint-Pol A, Yelamos B, Amessou M, Mills IG, Dugast M, Tenza D, Schu P, Antony C, McMahon HT, Lamaze C, Johannes L (2004) Clathrin adaptor epsinR is required for retrograde sorting on early endosomal membranes. Developmental cell 6 (4):525-538

Sandvig K, Skotland T, van Deurs B, Klokk TI (2013) Retrograde transport of protein toxins through the Golgi apparatus. Histochemistry and cell biology. doi:10.1007/s00418-013-1111Z

Sandvig K, van Deurs B (2000) Entry of ricin and Shiga toxin into cells: molecular mechanisms and medical perspectives. EMBO J 19 (22):5943-5950

Scarff KL, Judd LM, Toh BH, Gleeson PA, van Driel IR (1999) Gastric H(+),K(+)-adenosine triphosphatase beta subunit is required for normal function, development, and membrane structure of mouse parietal cells. Gastroenterology 117 (3):605-618

Scott GK, Fei H, Thomas L, Medigeshi GR, Thomas G (2006) A PACS-1, GGA3 and CK2 complex regulates CI-MPR trafficking. EMBO J 25 (19):4423-4435

Seaman MN (2007) Identification of a novel conserved sorting motif required for retromermediated endosome-to-TGN retrieval. J Cell Sci 120 (Pt 14):2378-2389

Sharpe HJ, Stevens TJ, Munro S (2010) A comprehensive comparison of transmembrane domains reveals organelle-specific properties. Cell 142 (1):158-169. doi:S0092-8674(10)00612-4 [pii] $\underline{10.1016 / j . c e l l .2010 .05 .037 ~}$

Shewan AM, van Dam EM, Martin S, Luen TB, Hong W, Bryant NJ, James DE (2003) GLUT4 recycles via a trans-Golgi network (TGN) subdomain enriched in Syntaxins 6 and 16 but not TGN38: involvement of an acidic targeting motif. Mol Biol Cell 14 (3):973-986

Shi A, Sun L, Banerjee R, Tobin M, Zhang Y, Grant BD (2009) Regulation of endosomal clathrin and retromer-mediated endosome to Golgi retrograde transport by the J-domain protein RME8. EMBO J 28 (21):3290-3302. doi:emboj2009272 [pii] 10.1038/emboj.2009.272

Tabuchi M, Yanatori I, Kawai Y, Kishi F (2010) Retromer-mediated direct sorting is required for proper endosomal recycling of the mammalian iron transporter DMT1. Journal of Cell Science 123 (Pt 5):756-766. doi:10.1242/jcs.060574 
Tai G, Lu L, Wang TL, Tang BL, Goud B, Johannes L, Hong W (2004) Participation of the syntaxin 5/Ykt6/GS28/GS15 SNARE complex in transport from the early/recycling endosome to the trans-Golgi network. Mol Biol Cell 15 (9):4011-4022

Thomas G (2002) Furin at the cutting edge: from protein traffic to embryogenesis and disease. Nat Rev Mol Cell Biol 3 (10):753-766. doi:10.1038/nrm934 nrm934 [pii]

Tran TH, Zeng Q, Hong W (2007) VAMP4 cycles from the cell surface to the trans-Golgi network via sorting and recycling endosomes. J Cell Sci 120 (Pt 6):1028-1041

Utskarpen A, Slagsvold HH, Iversen TG, Walchli S, Sandvig K (2006) Transport of ricin from endosomes to the Golgi apparatus is regulated by Rab6A and Rab6A'. Traffic 7 (6):663-672

Wan L, Molloy SS, Thomas L, Liu G, Xiang Y, Rybak SL, Thomas G (1998) PACS-1 defines a novel gene family of cytosolic sorting proteins required for trans-Golgi network localization. Cell 94 (2):205-216

Wang Y, Tai G, Lu L, Johannes L, Hong W, Tang BL (2005) Trans-Golgi network syntaxin 10 functions distinctly from syntaxins 6 and 16. Molecular membrane biology 22 (4):313-325

Wassmer T, Attar N, Harterink M, van Weering JR, Traer CJ, Oakley J, Goud B, Stephens DJ, Verkade P, Korswagen HC, Cullen PJ (2009) The retromer coat complex coordinates endosomal sorting and dynein-mediated transport, with carrier recognition by the trans-Golgi network. Developmental cell 17 (1):110-122

Wersto RP, Chrest FJ, Leary JF, Morris C, Stetler-Stevenson MA, Gabrielson E (2001) Doublet discrimination in DNA cell-cycle analysis. Cytometry 46 (5):296-306. doi:10.1002/cyto.1171 [pii]

Xu Y, Martin S, James DE, Hong W (2002) GS15 forms a SNARE complex with syntaxin 5, GS28, and Ykt6 and is implicated in traffic in the early cisternae of the Golgi apparatus. Mol Biol Cell 13 (10):3493-3507

Yadav S, Puri S, Linstedt AD (2009) A primary role for Golgi positioning in directed secretion, cell polarity, and wound healing. Mol Biol Cell 20 (6):1728-1736. doi:E08-10-1077 [pii] 10.1091/mbc.E08-10-1077

Yano H, Yamamoto-Hino M, Abe M, Kuwahara R, Haraguchi S, Kusaka I, Awano W, KinoshitaToyoda A, Toyoda H, Goto S (2005) Distinct functional units of the Golgi complex in Drosophila cells. Proc Natl Acad Sci U S A 102 (38):13467-13472. doi:0506681102 [pii] 10.1073/pnas.0506681102 


\section{Legends to Figures}

Figure 1 Endosome-to-TGN retrograde transport pathways

Proteins are internalized from the plasma membrane to the early endosomes, where cargo (red boxes) can undergo retrograde transport back to the TGN via a number of different pathways. Shiga toxin B (STxB) utilize the recycling endosome to TGN route, TGN38 utilizes the early endosome to TGN route whereas mannose-6-phosphate receptors (MPRs) and furin use the late endosome to TGN route.

\section{Figure 2 Golgi stacks are highly dispersed throughout the cytoplasm of parietal cells}

Gastric cell populations were isolated from the stomachs of wildtype mice and cultured for $24 \mathrm{~h}$ in gland culture media containing ranitidine (ref). Cultured cells were fixed in $1 \%$ formaldehyde, permeabilized, and with TRITC-conjugated phalloidin, mouse anti-GM130 antibodies followed by FITC-conjugated to anti-mouse IgG and rabbit anti-GRASP65 antibodies followed by ALEXA 647conjugated to anti-rabbit IgG. Parietal cells are identified by the presence of actin-rich intracellular secretory canaliculi vacuoles stained by TRITC-conjugated phalloidin (blue). The plasma membrane of the parietal cell is highlighted by the broken line. The images in (B) show the magnification of the boxed region in A. White arrows indicate double-labelled Golgi stacks. The bars represent $10 \mu \mathrm{m}$.

\section{Figure 3 Flow cytometry pulse width can be used for quantitation of cargo localization}

(A) The fluorescence signal of a fluorescent particle or cell as it moves through the detector of a flow cytometer. The maximum height of the signal is the pulse height, and the pulse width is a measure of the return of the signal to a basal level. The total area under the curve is the pulse area. (B) The different pulse width shapes resulting from either a concentrated small area of intracellular fluorescence or cell surface fluorescence. 
Fig. 1

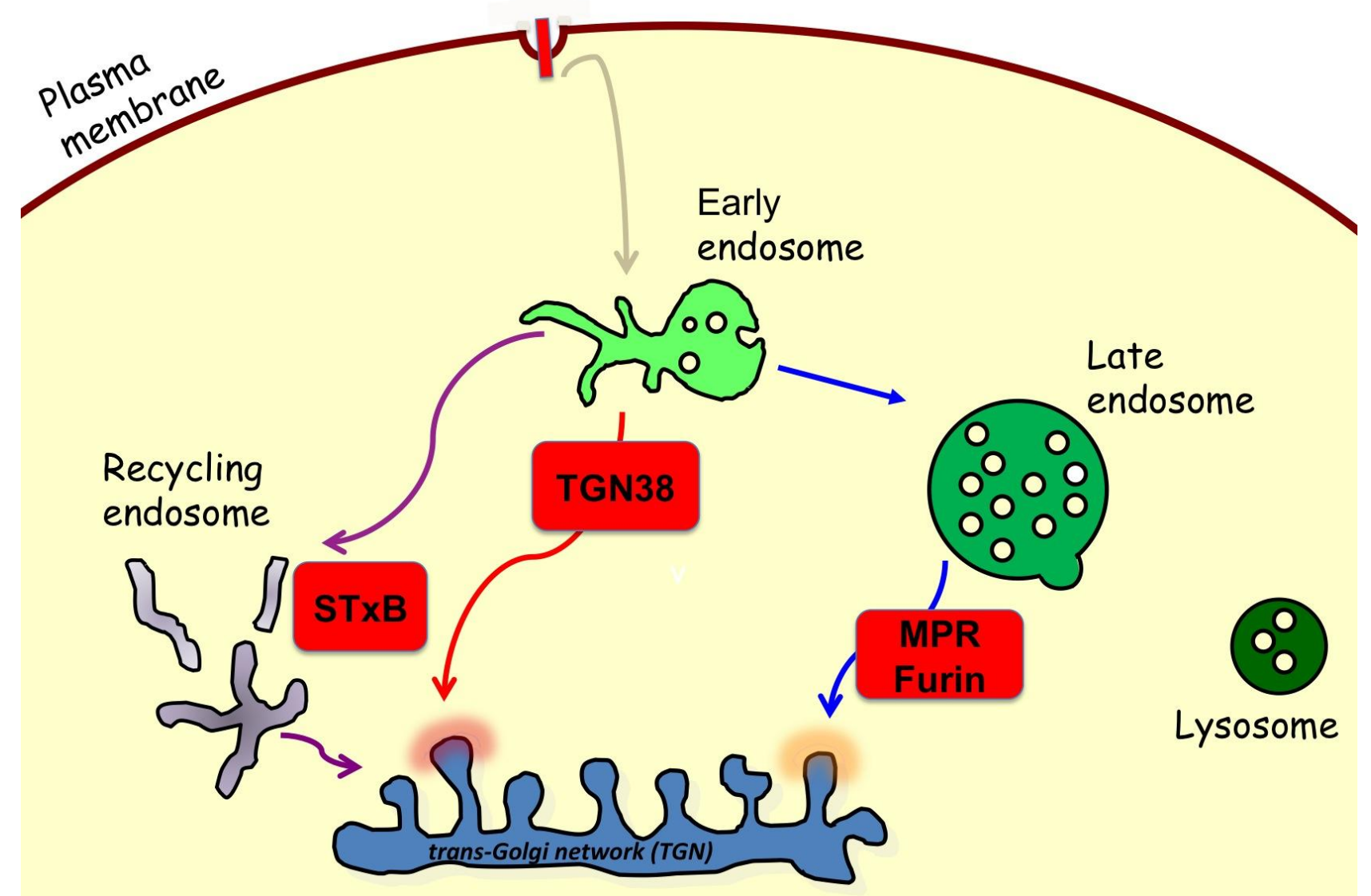


Fig. 2

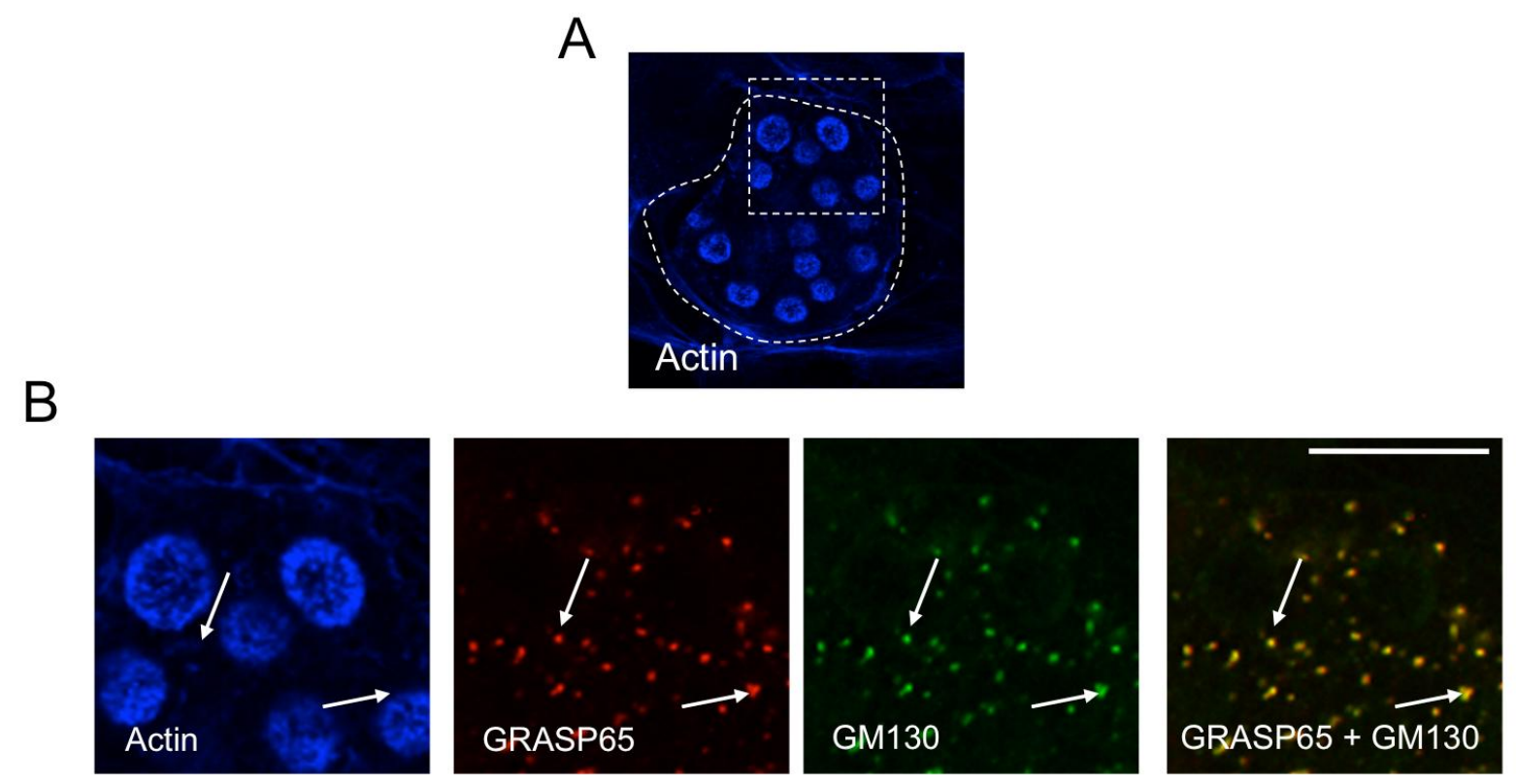


Fig. 3

A

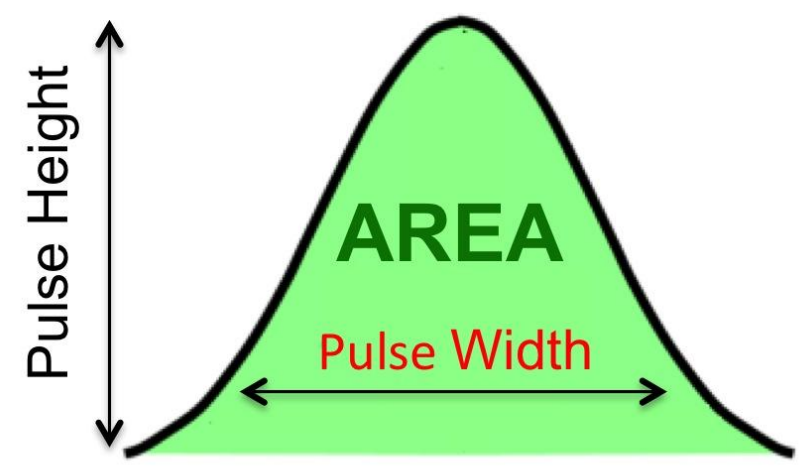

B

Fluorescence Patterns

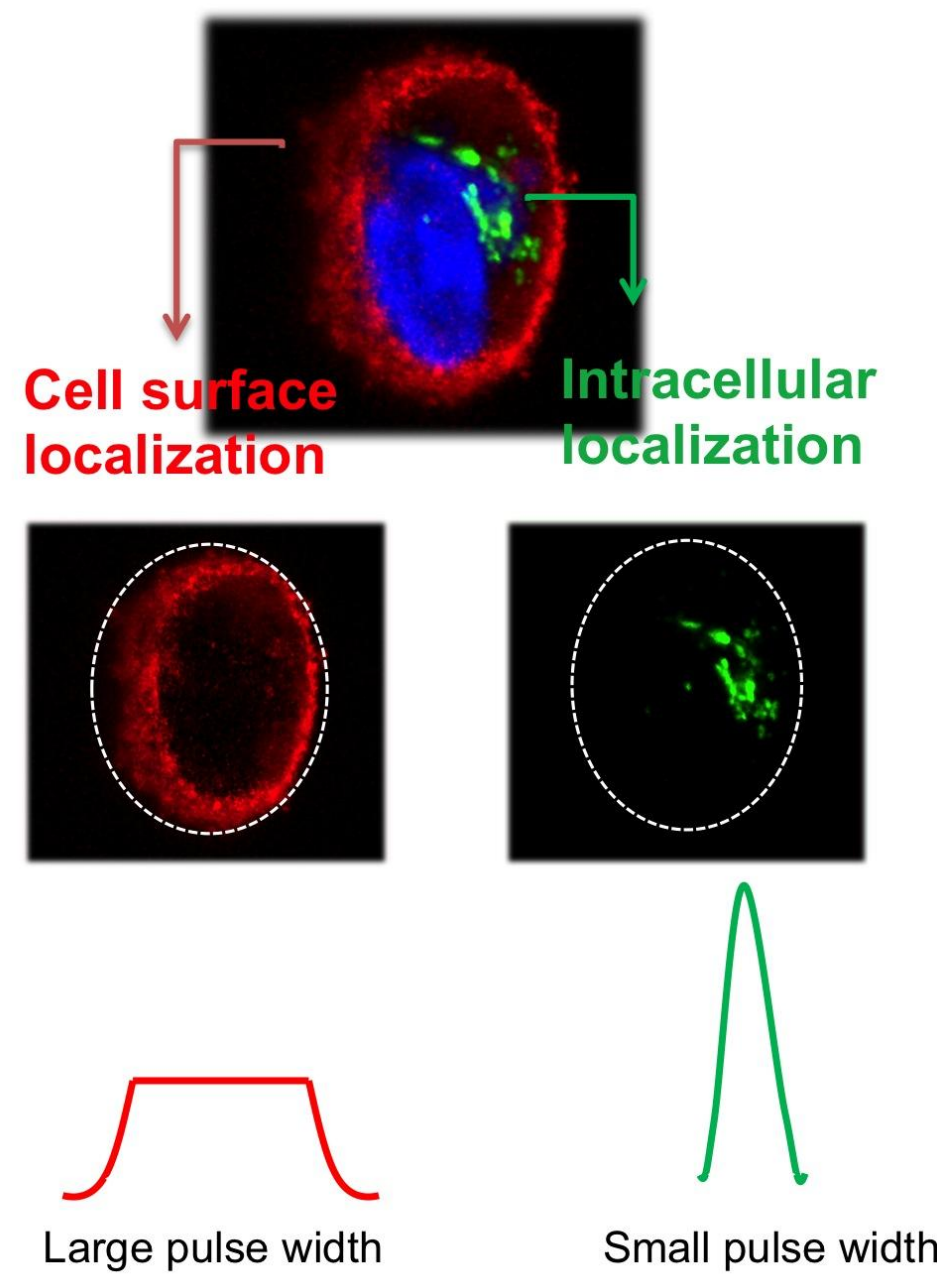

